Meta

Journal des traducteurs

Translators' Journal

\title{
Emploi de termes hybrides gréco-latins dans le langage médical
}

\section{Serge Guérin}

Volume 46, numéro 1, mars 2001

Traduction médicale et documentation / Medical translation and documentation

URI : https://id.erudit.org/iderudit/003886ar

DOI : https://doi.org/10.7202/003886ar

Aller au sommaire du numéro

Éditeur(s)

Les Presses de l'Université de Montréal

ISSN

0026-0452 (imprimé)

1492-1421 (numérique)

Découvrir la revue

Citer cet article

Guérin, S. (2001). Emploi de termes hybrides gréco-latins dans le langage médical. Meta, 46(1), 7-15. https://doi.org/10.7202/003886ar

\section{Résumé de l'article}

De nombreux termes médicaux ont été formés en transgressant une règle étymologique classique, c'est-à-dire en mélangeant des éléments grecs et latins. Nous avons colligé près de 250 de ces hybrides gréco-latins en français médical et constaté qu'une dizaine sont en concurrence avec un synonyme étymologiquement homogène ; la moitié au moins connaissent un emploi quelque peu différent de leur " équivalent " plus " pur ", alors que les autres n'ont pas d'équivalent usité de la sorte. Un phénomène analogue est observé en anglais médical, bien que nous n'ayons pas été en mesure de le quantifier de la même façon. Il paraît somme toute illusoire de vouloir bannir les hybrides du vocabulaire médical, sauf éventuellement en créant des mots nouveaux. On peut même voir à ces hybrides une certaine utilité, en dehors du simple fait qu'il sont passés dans l'usage. Certains, en effet, permettent de marquer une nuance, d'une manière arbitraire mais commode. En outre, pour celui ou celle qui étudie la terminologie médicale, l'apprentissage des hybrides gréco-latins peut constituer un moyen efficace de mémoriser l'étymologie.
Ce document est protégé par la loi sur le droit d'auteur. L’utilisation des services d’Érudit (y compris la reproduction) est assujettie à sa politique d'utilisation que vous pouvez consulter en ligne.

https://apropos.erudit.org/fr/usagers/politique-dutilisation/ 


\title{
Emploi de termes hybrides gréco-latins dans le langage médical
}

\author{
SERGE QUÉRIN \\ Université de Montréal, Montréal, Canada
}

\begin{abstract}
RÉSUMÉ
De nombreux termes médicaux ont été formés en transgressant une règle étymologique classique, c'est-à-dire en mélangeant des éléments grecs et latins. Nous avons colligé près de 250 de ces hybrides gréco-latins en français médical et constaté qu'une dizaine sont en concurrence avec un synonyme étymologiquement homogène; la moitié au moins connaissent un emploi quelque peu différent de leur «équivalent» plus «pur», alors que les autres n'ont pas d'équivalent usité de la sorte. Un phénomène analogue est observé en anglais médical, bien que nous n'ayons pas été en mesure de le quantifier de la même façon. Il paraît somme toute illusoire de vouloir bannir les hybrides du vocabulaire médical, sauf éventuellement en créant des mots nouveaux. On peut même voir à ces hybrides une certaine utilité, en dehors du simple fait qu'il sont passés dans l'usage. Certains, en effet, permettent de marquer une nuance, d'une manière arbitraire mais commode. En outre, pour celui ou celle qui étudie la terminologie médicale, l'apprentissage des hybrides gréco-latins peut constituer un moyen efficace de mémoriser l'étymologie.
\end{abstract}

\section{ABSTRACT}

A large number of medical terms have been formed without regard for a classic etymological rule, i.e. mixing up Greek and Latin elements. We have identified close to 250 of these Greco-Latin hybrids in medical French and have noted that ten have an etymologically homogeneous synonym, half as much are used somewhat differently than their "pure" "counterpart", while the others have no such equivalent in use. A similar phenomenon occurs in medical English, although we have been unable to quantify it in the same way. Finally, the goal of banning the use of hybrids from the medical vocabulary appears illusive, except perhaps when creating new words. Hybrids may even be useful, apart from the mere fact that they already are in common use. As a matter of fact, some of them introduce a slight difference of meaning, in an arbitrary but convenient way. Moreover, for the student of medical terminology, learning greco-latin hybrids may be an effective method to memorize etymology.

\section{MOTS-CLÉS/KEYWORDS}

hybrides gréco-latins, bases de données, terminologie médicale, français, anglais

Une règle classique — d'aucuns diront arbitraire, ou à tout le moins archaïque — veut que les mots doivent être homogènes d'un point de vue étymologique, c'est-à-dire, dans le cas des termes médicaux, être formés uniquement d'éléments grecs ou d'éléments latins. N'en déplaise aux puristes de l'étymologie, cette règle est fréquemment transgressée, autant dans le vocabulaire courant que dans celui de la médecine. Des «hybrides gréco-latins» existent, en fait, tant en anglais qu'en français médical (Van Hoof 1997) mais, à notre connaissance, aucune étude systématique ne leur a encore été consacrée. Il existe aussi des hybrides formés, par exemple, de la combinaison d'un élément anglais et d'un élément grec (to scan + graphein $\varnothing$ scanographie), ou 
arabe et grec (al-qilyi + -osis $\varnothing$ alcalose), ou encore d'un éponyme et d'un élément grec (Tulare, Californie + haima $\varnothing$ tularémie). Cependant, leur nombre est extrêmement faible comparativement aux hybrides gréco-latins et ils ne seront pas davantage envisagés ici.

\section{MÉTHODES}

\section{Établissement de listes de termes médicaux hybrides}

Les listes de termes médicaux hybrides des tableaux 1 à 3 ont été dressées dans un premier temps à partir de quelques relevés partiels déjà publiés (Skinner 1961:216; Roberts 1966: 13-14; Hussey 1976; Dirckx 1977; Bouché 1984: 241-252; Van Hoof 1997: 77-78; Quérin 1998: 96; Quérin 2000: 6), puis en parcourant le Dictionnaire de médecine Flammarion muni des listes de préfixes et suffixes médicaux grecs et latins établies par Bouché (1994: 12-79, 145-167). Les listes ont ensuite été complétées en vérifiant chacun des suffixes relevés par Bouché à l'aide du moteur de recherche du Petit Robert sur cédérom (1996), à défaut d'un outil électronique semblable jumelé à un dictionnaire médical. Sauf erreur, les pseudo-hybrides composés d'un élément grec et d'un autre passé du grec au latin n'ont pas été retenus.

\section{Analyse de la fréquence relative des hybrides gréco-latins ayant un synonyme usité étymologiquement homogène et des termes anglais correspondants}

La fréquence - c'est-à-dire le nombre d'occurrences — de chacun des termes du tableau 1 a été vérifiée dans la banque d'articles en français de l'Institut de l'information scientifique et technique (INIST) 1990-2000 dans le cas des termes français, et dans Medline pour la période allant de 1997 à mai 2000 pour les termes anglais. Ont été répertoriés dans ce tableau uniquement les termes français hybrides qui ont été retrouvés dans la banque de l'INIST tout autant que leur équivalent étymologiquement homogène, dans un rapport de fréquence d'au moins 1 pour 4 . Tout comme dans Medline pour les articles en anglais, les recherches dans la banque de l'INIST ont porté à la fois sur les titres et résumés d'articles rédigés en français, en faisant appel au mode de consultation expert et à l'équation: «terme»< <N $>$ (title, abstract). Certaines interrogations de la banque de l'INIST ont été accidentellement écourtées par le moteur de recherche, mais le nombre de documents explorés a avoisiné 3000000 pour chacun des termes. Enfin, les paires de termes nocturia-nycturia et afebrile-apyretic ont fait l'objet d'une interrogation spéciale dans Medline, de même que les termes correspondants en français dans la banque de l'INIST.

\section{Hybrides gréco-latins dont l'emploi diffère de celui de leur "équivalent» étymologiquement homogène}

Les nuances d'emploi entre chacun des hybrides répertoriés au tableau 2 et leur «équivalent » étymologiquement homogène ont d'abord été repérées dans la banque de l'INIST. Les éventuels écarts entre l'usage ainsi observé et les définitions proposées par trois dictionnaires (Flammarion, Garnier-Delamare et celui de Manuila et al. en 4 volumes) ont ensuite été relevés. 


\section{Hybrides gréco-latins d'usage courant en français médical n'ayant pas d'équivalent usité étymologiquement homogène}

D'autres termes hybrides, n'ayant pas d'équivalent usité étymologiquement homogène, ont aussi fait l'objet d'un relevé systématique (tableau 3). Ils ont été classés en deux colonnes, avec à chaque ligne du tableau un terme type choisi arbitrairement à gauche, et éventuellement d'autres hybrides ayant le même préfixe ou suffixe à droite.

\section{RÉSULTATS}

Le tableau 1 donne la liste d'une dizaine d'hybrides gréco-latins qui sont couramment employés bien qu'ils soient en concurrence avec un synonyme étymologiquement homogène. Certains hybrides sont plus fréquents que leur synonyme (adipocyte, antifongique, cancérogène, lithotritie, rectite), alors que d'autres le sont moins (mammectomie, myringoplastie, quadriplégie). Les hybrides correspondants en anglais sont moins employés (seuls adipocyte et antifungal l'emportent en fréquence sur leur synonyme respectif), mais il faut souligner qu'ils ne représentent qu'une partie seulement de tous les hybrides gréco-latins employés en anglais médical. Ainsi, nocturia, hybride, est quasi constant en anglais, alors que nycturia est à peu près inconnu (131 occurrences du premier dans Medline, contre 1 seule pour le second). C'est l'inverse en français: nycturie est courant, mais nocturie est tout à fait inusité, aucun emploi de ce terme n'ayant été identifié dans la banque de l'INIST, ce qui explique son absence du tableau 1 . De même, alors que l'adjectif hybride afebrile est d'usage courant en anglais (163 occurrences, contre seulement 2 pour apyretic), en français, au contraire, afébrile est à peu près inconnu (1 seule occurrence au cours de la période de dix ans que nous avons étudiée), au profit de apyrétique.

Certains hybrides connaissent un emploi quelque peu différent de celui de leur «équivalent» étymologiquement homogène (tableau 2). Les deux termes de chaque paire du tableau 2 ne sont donc pas en concurrence, contrairement à ceux du tableau 1. Il est à noter que nous n'avons relevé dans le tableau 2 que les nuances respectées par l'usage actuel tel que reflété dans la banque de l'INIST. C'est pourquoi, par exemple, la distinction classique entre adénocancer et adénocarcinome n'a pas été relevée dans le tableau, puisqu'elle n'a pas été retrouvée dans les articles explorés.

Enfin, le tableau 3 montre que la grande majorité des hybrides gréco-latins employés en médecine n'ont pas d'équivalent usité étymologiquement homogène, ce que nous avons vérifié dans la banque de données de l'INIST dans les cas a priori les plus plausibles.

Les listes d'hybrides gréco-latins médicaux des tableaux 1 à 3 sont évidemment incomplètes, compte tenu de la méthode de repérage utilisée, mais elles donnent un bon aperçu de la variété de termes de ce genre dans le vocabulaire médical. Au total, 249 termes ont été répertoriés dans les trois tableaux, ce qui indique bien la fréquence des hybrides gréco-latins en médecine. Alors que certains préfixes et suffixes entrent dans la formation d'un très grand nombre d'hybrides (en particulier -algie, anti-, -ectomie et -tomie, -gène, -graphie, hyper- et hypo-), d'autres, pourtant très fréquents en médecine, ne semblent jamais avoir servi à la formation de tels termes (par exemple, le préfixe d'origine grecque ana-). 


\section{DISCUSSION}

À la lumière de ce travail, il est malheureusement impossible d'énoncer une opinion quant à la fréquence relative des hybrides gréco-latins en français et en anglais médical. On peut simplement dire qu'ils sont fréquents dans les deux langues, en dépit de la coexistence d'un synonyme étymologiquement homogène dans certains cas.

Une autre limite de cette étude est le fait que beaucoup d'articles répertoriés dans Medline ont pu avoir été écrits en anglais, sans révision, par des auteurs ayant une autre langue maternelle. Il est toutefois peu probable que ce facteur ait influencé de manière importante la fréquence d'emploi de certains hybrides par rapport à leur équivalent étymologiquement homogène.

Ce travail permet de constater qu'il est illusoire de vouloir se défaire des hybrides gréco-latins déjà présents dans le vocabulaire médical. On peut, au nom de la règle classique, proposer de les éviter en créant des mots nouveaux. Cependant, à l'évidence, les anticorps, les transaminases et les varicocèles qui garnissent le lexique médical depuis belle lurette sont là pour rester, tout comme d'ailleurs le mot automobile dans le langage courant. Il faut aussi accepter mammographie tout autant que mastectomie. De plus, on l'a vu dans le cas, par exemple, des reins multikystiques et polykystiques, certains hybrides permettent de marquer une nuance, d'une manière certes arbitraire et irrespectueuse de l'étymologie, mais néanmoins bien commode.

Une question viendra sans doute à l'esprit du traducteur: que doit-on penser de l'emploi occasionnel en français de rares hybrides moins fréquents que leur équivalent étymologiquement homogène alors que c'est l'inverse que l'on constate dans le cas des termes anglais correspondants. On pense en particulier à l'adjectif hybride afébrile, qui est inusité en français, même s'il est donné comme synonyme d'apyrétique dans les dictionnaires de Manuila et al. (1970-1975, 1996), alors qu'afebrile est tout à fait courant en anglais. Afébrile doit-il par conséquent être considéré comme un anglicisme? La réponse est sans doute oui, d'autant plus que, dans le français médical parlé, le mot est courant au Québec et en Belgique (sans doute en raison de contacts plus fréquents avec l'anglais) alors qu'il est à peu près inconnu en France (D. Erbilgin, communication personnelle).

Deux dernières remarques paraissent à propos. D’abord, les lecteurs intéressés à l'enseignement de la terminologie médicale auront peut-être constaté que l'apprentissage des hybrides, du fait qu'il oppose le grec et le latin, est un bon moyen mnémotechnique d'acquérir des notions d'étymologie. Ensuite, ceux orientés vers la recherche terminologique auront sans doute vu dans notre travail un exemple de l'utilité de banques de données comme celle de l'INIST et Medline. Ce dernier constat est bien en accord avec le thème de ce numéro spécial de Meta, «Traduction médicale et documentation».

\section{RÉFÉRENCES}

Aronson, J. K. (2000) : “Where Name and Image Meet' - The Argument for 'Adrenaline,"” British Medical Journal, 320, pp. 506-509.

Bouché, P. (1984): Les mots de la médecine, Paris, Belin.

Dictionnaire de médecine Flammarion (1994), $5^{\mathrm{e}}$ éd., Paris, Flammarion, coll. "Médecine Sciences».

Dictionnaire de termes de médecine Garnier-Delamare (1992), 23e éd., Paris, Maloine.

Dirckx, J. H. (1977): "Hybrid Words in Medical Terminology," Journal of the American Medical Association, 238, pp. 2043-2045. 
Dubois, J., H. Mitterand et A. Dauzat (1998): Dictionnaire étymologique et historique du français, Paris, Larousse.

Hall, F. M. (1999): “What's in a Name - Revisited," American Journal of Roentgenology, 173, p. 850.

Hussey, H. H. (1976) : "Word Origins," Journal of the American Medical Association, 236, pp. 22122213.

Institut De L'information scientifique Et Technique (2000): Article@INIST, <http:// form.inist.fr/public/fre/conslt.htm> (6 septembre 2000).

Manuila, A. et L., M. Nicole et M. Lambert (1970-1975): Dictionnaire français de médecine et de biologie, Paris, Masson.

Manuila, A. et L., et M. Nicoulin (1996): Dictionnaire médical, Paris, Masson.

Medline, version 7.8 Millenium [cédérom], New York, Ovid Technologies Inc., mai 2000

QuérIN, S. (1998): Dictionnaire des difficultés du français médical, Saint-Hyacinthe, Edisem.

— (2000): «À propos du vocabulaire néphrologique», Néphrologie, 21, p. 5-7.

Roberts, F. (1966): Medical Terms: Their Origin and Construction, London, Heinemann.

Schillinger, F., R. Montagnac, T. Milcent et D. Schillinger (1988): «Maladie multikystique acquise (MMA) chez le dialysé chronique», Néphrologie, 9, p. 105-108.

Skinner, H. A. (1961): The Origin of Medical Terms, Baltimore, Williams \& Wilkins.

VAN Hoof, H. (1997): «Le langage de la médecine: étude comparative de l'anglais et du français ", Le langage et l'homme, 34, p. 75-91.

\section{ANNEXES}

Tableau 1 : Hybrides gréco-latins d'usage courant en français médical ayant un synonyme usité étymologiquement homogène, avec les termes anglais correspondants et le nombre d'occurrences de chaque terme dans les titres et résumés des articles des banques de données de l'INIST 1990-2000, en français, et Medline 1997-2000, en anglais.

\begin{tabular}{|c|c|c|c|}
\hline \multicolumn{2}{|c|}{ Termes français } & \multicolumn{2}{|c|}{ Termes anglais } \\
\hline Hybride & Homogène & Hybride & Homogène \\
\hline Adipocyte (20) & Lipocyte (2) & Adipocyte (786) & Lipocyte (10) \\
\hline Antifongique (80) & Antimycotique (2) & Antifungal (1741) & Antimycotic (123) \\
\hline $\begin{aligned} & \text { Cancérigène (18) } \\
& \text { - cancérogène }{ }^{\mathrm{a}}(38)\end{aligned}$ & Carcinogène (23) & $\begin{array}{c}\text { Cancerigenic (1) } \\
\text { - cancerogenic (7) }\end{array}$ & Carcinogenic (1700) \\
\hline Cancérologie (217) & Oncologie (225) & Cancerology (4) & Oncology (2896) \\
\hline Homolatéral (19) & Ipsilatéral (15) & Homolateral (60) & Ipsilateral (3919) \\
\hline Lithotritie $^{\text {b }}(41)$ & Lithotripsie ${ }^{\mathrm{b}}(10)$ & Lithotrity (2) & Lithotripsy (502) \\
\hline Mammectomie (17) & Mastectomie (50) & Mammectomy (0) & Mastectomy (784) \\
\hline Myringoplastie (3) & Tympanoplastie (7) & Myringoplasty (28) & Tympanoplasty (85) \\
\hline Rectite (15) & Proctite (3) & Rectitis (1) & Proctitis (113) \\
\hline Quadriplégie (8) & Tétraplégie (31) & Quadriplegia (123) & Tetraplegia (180) \\
\hline
\end{tabular}

Notes:

a. L'Académie des sciences de France recommande cancérogène.

b. Malgré leur ressemblance, les termes lithotritie et lithotriptie sont construits quelque peu différemment, le premier faisant appel au tritor latin alors que le second fait plutôt intervenir le triptêr grec. Lithotriteur se distingue de la même façon de lithotripteur.

Les termes les plus couramment utilisés (> 60\% des cas pour une paire donnée) sont indiqués en caractères gras.

Sources: Bouché 1994: 243-252; Dubois et al. 1995; INIST 2000; Manuila et al. 1996; Medline 2000; Quérin 1998 : 96; Quérin 2000: 6; Van Hoof 1997: 77-78. 
Tableau 2: Hybrides gréco-latins dont l'emploi diffère de celui de leur "équivalent » étymologiquement homogène.

\begin{tabular}{|c|c|c|c|}
\hline \multicolumn{2}{|c|}{ Hybride } & \multicolumn{2}{|c|}{ «Équivalent» } \\
\hline $\begin{array}{c}\text { Terme } \\
\text { Audiométrie }\end{array}$ & $\begin{array}{l}\text { Emploi(s) } \\
\text { particulier(s) } \\
\text { Mesure précise } \\
\text { de l'audition a }\end{array}$ & $\begin{array}{l}\text { Terme } \\
\text { Acoumétrie }\end{array}$ & $\begin{array}{c}\text { Emploi(s) } \\
\text { particulier(s) } \\
\text { Estimation approximative } \\
\text { et subjective } \\
\text { de l'audition }{ }^{\text {b }}\end{array}$ \\
\hline Endoveineux(se) & $\begin{array}{l}\text { Se dit en général d'un } \\
\text { dispositif mis en place à } \\
\text { demeure dans une veine } \\
\text { (électrode, clip, filtre) } \\
\text { ou } \\
\text { de ce qui est relatif } \\
\text { à l'endoveine c }\end{array}$ & Intraveineux(se) & $\begin{array}{c}\text { Se dit d'une injection faite } \\
\text { (ou d'une substance } \\
\text { administrée) } \\
\text { dans une veine } \\
\text { ou } \\
\text { de ce qui concerne } \\
\text { l'intérieur d'une veine }\end{array}$ \\
\hline Nucléolyse & $\begin{array}{l}\text { Dissolution du noyau } \\
\text { pulpeux d'un disque } \\
\text { intervertébral par injection } \\
\text { d'une enzyme protéolytique }\end{array}$ & Caryolyse & $\begin{array}{l}\text { Destruction par dissolution } \\
\text { du noyau d'une cellule }\end{array}$ \\
\hline $\begin{array}{c}\operatorname{Rein}(s) \\
\text { multikystique(s) }\end{array}$ & $\begin{array}{c}\text { Affection congénitale, } \\
\text { gén. unilatérale } \\
\text { ou } \\
\text { affection acquise, bilatérale, } \\
\text { résultant d'une } \\
\text { insuffisance rénale } \\
\text { (maladie kystique acquise } \\
\text { du dialysé) }\end{array}$ & $\begin{array}{l}\text { Reins polykystiques } \\
\text { (ou polykystose } \\
\text { rénale) }\end{array}$ & $\begin{array}{l}\text { Affection héréditaire, } \\
\text { bilatérale, responsable } \\
\text { d'une insuffisance rénale }\end{array}$ \\
\hline Rénogramme ${ }^{\mathrm{e}}$ & $\begin{array}{c}\text { Courbe d'excrétion rénale } \\
\text { d'une substance } \\
\text { isotopique }\end{array}$ & Néphrogramme & $\begin{array}{l}\text { Image du parenchyme } \\
\text { rénal obtenue après } \\
\text { injection d'une substance } \\
\text { de contraste radiologique } \\
\text { ou d'un isotope radioactif }\end{array}$ \\
\hline
\end{tabular}

Notes:

a. L'audiogramme, autre terme hybride, est la représentation graphique de l'audition ainsi mesurée.

b. Cette définition est celle du dictionnaire Flammarion. Les auteurs du Garnier-Delamare et du Manuila sont plutôt d'avis que acoumétrie est un terme générique pour désigner une forme quelconque d'évaluation quelconque de l'audition, y compris l'audiométrie. Dans une acception comme dans l'autre, acoumétrie n'est pas synonyme de audiométrie.

c. Au Québec, on parle souvent de "pyélographie endoveineuse» ou même simplement, en jargon médical, d' «endoveineuse» tout court pour désigner une urographie intraveineuse. L'emploi du terme pyélographie, inspiré de l'anglais pyelography, est lui-même étonnant dans ce cas, puisqu'il s'agit bien d'un examen de l'ensemble de l'arbre urinaire. C'est sans doute le témoin d'une époque où les produits de contraste intraveineux ne permettaient de bien opacifier que le bassinet (puelos en grec) (Hall 1999).

d. On rencontre aussi l'expression rein plurikystique, qui est un autre hybride dont la définition n'est pas clairement établie, mais qui tend à désigner tout rein porteur de plusieurs kystes, sans qu'il s'agisse clairement ni d'un rein multikystique, ni d'un rein polykystique.

e. Parfois appelé néphrogramme isotopique.

Sources : Bouché 1994: 244, 249; Dictionnaire de médecine Flammarion 1994; Dictionnaire des termes de médecine Garnier-Delamare 1992; Manuila et al. 1970-75; Le petit Larousse 1999; Quérin 2000: 6; Schillinger et al. 1988. 
Tableau 3: Hybrides gréco-latins d'usage courant en français médical n'ayant pas d'équivalent usité étymologiquement homogène

\begin{tabular}{|c|c|}
\hline Cas types & Autres hybrides ayant le même préfixe ou suffixe \\
\hline Adrénergique ${ }^{a}$ & Adrénolytique \\
\hline Albuminurie & Bacillurie, calciurie, cupriurie, fécalurie \\
\hline \multicolumn{2}{|l|}{ Angiotensine } \\
\hline Annuloplastie & Annulocyte \\
\hline \multicolumn{2}{|l|}{ Antéhypophyse } \\
\hline Anticorps & $\begin{array}{c}\text { Antiacide, antiagrégant, antiangineux, anticancéreux, anticoagulant, } \\
\text { anticonceptionnel, anticonvulsivant, antidépresseur, antiglobuline, } \\
\text { antihypertenseur, anti-inflammatoire }{ }^{b} \text {, antipaludique, antiprurigineux, } \\
\text { antirabique, antisens, antisérum, antitussif, antiviral }\end{array}$ \\
\hline Anxiolytique & Apicolyse, fibrinolyse \\
\hline Appendicectomie & $\begin{array}{c}\text { Antrectomie, cellulectomie, duodénectomie, omentectomie }{ }^{a} \text {, } \\
\text { ovariectomie }^{a} \text {, patellectomie, scapullectomie, valvulectomie, } \\
\text { vasectomie, vésiculectomie }\end{array}$ \\
\hline Atriotomie & Atriomégalie \\
\hline Auto-immunité & Autoclave, automutilation, autotransfusion \\
\hline Barorécepteur & Chémorécepteur \\
\hline Bi-acromial & Biballisme, bi-ischiatique \\
\hline \multicolumn{2}{|l|}{ Biodisponibilité } \\
\hline Bronchodilatation & Bronchoconstriction \\
\hline Calcémie & Cuprémie, plombémie, septicémie, volémie \\
\hline \multicolumn{2}{|l|}{ Campimétrie } \\
\hline \multicolumn{2}{|l|}{ Cellulite } \\
\hline Cervicarthrose & Dorsarthrose, lombarthrose, coxarthrose \\
\hline Claustrophobie & Cancérophobie \\
\hline Coagulopathie & Coronaropathie, fotopathie, tubulopathie \\
\hline Commissurotomie & Duodénotomie, lombotomie, septotomie, sinusotomie, valvulotomie \\
\hline \multicolumn{2}{|l|}{ Coproculture $^{a}$} \\
\hline Corticothérapie & Insulinothérapie, sérothérapie \\
\hline Cryoglobuline & Macroglobuline \\
\hline Dextrocardie & Lévocardie \\
\hline \multicolumn{2}{|l|}{ Extrasystole } \\
\hline Fibrinogène & $\begin{array}{l}\text { Agglutinogène, anxiogène, hallucinogène, immunogène, } \\
\text { lactogène, mutagène }\end{array}$ \\
\hline Fibroblaste & Fibrocyte, fibromatose \\
\hline Fibrome & Fécalome, fibromyome, fibrosarcome \\
\hline Fibroscope ${ }^{c}$ & Anuscope, duodénoscope, fluoroscopie, négatoscope \\
\hline Glomérulonéphrite & Glomérulosclérose \\
\hline Granulocyte & Granulopénie, granulopoïse \\
\hline Hémoglobine & Hémagglutination, hémoculture, hémomédiastin, hémoperfusion \\
\hline
\end{tabular}


14 Meta, XLVI, 1, 2001

\begin{tabular}{|c|c|}
\hline Histocompatibilité & \\
\hline Hypertension & $\begin{array}{c}\text { Hypercoagulabilité, hypercorticisme, hyperinsulinisme, } \\
\text { hyperventilation, hyperviscosité }\end{array}$ \\
\hline \multicolumn{2}{|l|}{ Hypocomplémentémie ${ }^{d}$} \\
\hline Immunoélectrophorèse & Immunoblaste, immunocyte, immunoenzymatique, immunothérapie \\
\hline \multicolumn{2}{|l|}{ Interleukine } \\
\hline \multicolumn{2}{|l|}{ Intrathécal } \\
\hline Jéjunostomie & Duodénostomie \\
\hline Liposuccion $^{a}$ & Liposoluble \\
\hline Lombalgie & $\begin{array}{l}\text { Cervicalgie, coxalgie, cruralgie, dorsalgie, funiculalgie, } \\
\text { ovarialgie, précordialgie, pubalgie, scapulalgie, viscéralgie }\end{array}$ \\
\hline Mammographie ${ }^{a}$ & $\begin{array}{c}\text { Cavographie, coronarographie, scintigraphie, sinusographie, } \\
\text { spirographie, ventriculographie, vésiculographie }\end{array}$ \\
\hline \multicolumn{2}{|l|}{ Mégavessie } \\
\hline \multicolumn{2}{|l|}{ Mononucléose } \\
\hline Microorganisme & Microcornée, microponction, microtubule, microvillosité \\
\hline \multicolumn{2}{|l|}{ Mucolipidose } \\
\hline Myélofibrose & Myéloprolifératif \\
\hline Myoglobine & Myofibrille, myofilament, myorelaxant \\
\hline Neurofibrome & Neurofibrille, neurofilament \\
\hline Ovocyte & Ovogenèse. ovogonie \\
\hline Pharmacodépendance & Pharmacovigilance \\
\hline Photosensibilité & Photocoagulation \\
\hline Polynucléaire ${ }^{a e}$ & Polyglobulie, polyradiculonévrite \\
\hline \multicolumn{2}{|l|}{ Pseudobulbaire } \\
\hline Radiographie & Radiodermite, radiodiagnostic, radio-isotope \\
\hline Radiologie & Immunologie, infectiologie, sérologie, virologie \\
\hline Rectorragie & Rectocèle $^{a}$, rectopexie, rectorraphie, rectoscopie ${ }^{a}$ \\
\hline Réticulocyte & Réticulohistiocytome, réticulosarcome \\
\hline Rétinoblastome & Rétinocytome, rétinoépithéliome \\
\hline Rétrolisthésis & Rétrochéilie, rétrognathie \\
\hline Rhinovirus & Cytomégalovirus \\
\hline Sérodiagnostic & Séroprophylaxie \\
\hline Spirométrie & Tomodensitométrie \\
\hline Subictère & Subnarcose \\
\hline Transaminase & Insulinase \\
\hline Valvuloplastie & Septoplastie \\
\hline Varicocèle & Mucocèle \\
\hline Vasoplégie & \\
\hline
\end{tabular}

Notes:

a. Certains hybrides ont un équivalent étymologiquement homogène particulièrement plausible mais néanmoins inusité. Dans l'ordre d'apparition de ces hybrides dans le tableau, ces équivalents sont épinéphrinergique (Aronson 2000), épiploectomie, oophorectomie, fécoculture, adiposuccion, mastographie (alors que mastectomie est courant, cf. tableau 1), multinucléaire, proctocèle et proctoscopie. 
b. Antiphlogistique est un synonyme étymologiquement homogène de anti-inflammatoire, mais il est désuet.

c. Instrument fait de fibres optiques servant à examiner l'intérieur d'un organe (on dit mieux: endoscope).

d. La plupart des hybrides construits avec le préfixe hyper- (voir hypertension) ont un contraire construit avec hypo-, hybride lui aussi.

e. Outre le fait qu'il s'agit d'un hybride, le terme polynucléaire est impropre dans la mesure où il désigne une variété de leucocyte (aussi appelée granulocyte, autre terme hybride) qui ne possède, en réalité, qu'un seul noyau polysegmenté.

Chaque terme n'apparait qu'une fois dans le tableau. Les dérivés de termes hybrides (par exemple, hypercalcémie, hypotenseur, microalbuminurie), de même que les noms de médicaments et de microorganismes, ont été omis de ce relevé.

Sources: Bouché 1994: 243-252; Dubois et al. 1995; INIST 2000; Manuila et al. 1996; Medline 2000; Quérin 1998: 96; Quérin 2000: 6; Van Hoof 1997: 75, 80. 